\title{
Intensive Care Unit-Specific Virtual Reality for Psychological Recovery After ICU Treatment for COVID-19; A Brief Case Report
}

\author{
Johan H. Vlake ${ }^{1,2}$, Jasper van Bommel ${ }^{1}$, Merel E. Hellemons ${ }^{3}$, Evert-Jan Wils ${ }^{2}$, \\ Diederik Gommers ${ }^{1}$ and Michel E. van Genderen ${ }^{1,2 *}$ \\ ${ }^{1}$ Department of Intensive Care, Erasmus MC, Rotterdam, Netherlands, ${ }^{2}$ Department of Intensive Care, Franciscus Gasthuis \\ \& Vlietland, Rotterdam, Netherlands, ${ }^{3}$ Department of Pulmonology, Erasmus MC, Rotterdam, Netherlands
}

A substantial number of ICU survivors are expected due to the SARS-CoV-2 outbreak,

OPEN ACCESS

Edited by:

Marcelo Arruda Nakazone,

Faculty of Medicine of São José Do

Rio Preto, Brazil

Reviewed by:

Marco Di Nicola

Catholic University of the Sacred

Heart, Italy

Oscar Joseph Bienvenu,

Johns Hopkins Medicine,

United States

Leslie Hoffman,

University of Pittsburgh, United States

*Correspondence:

Michel E. van Genderen

m.vangenderen@erasmusmc.nl

Specialty section:

This article was submitted to

Intensive Care Medicine and

Anesthesiology,

a section of the journal

Frontiers in Medicine

Received: 13 November 2020 Accepted: 29 December 2020

Published: 05 February 2021

Citation:

Vlake JH, van Bommel J, Hellemons ME, Wils E-J, Gommers D

and van Genderen ME (2021)

Intensive Care Unit-Specific Virtual Reality for Psychological Recovery After ICU Treatment for COVID-19;

A Brief Case Report.

Front. Med. 7:629086.

doi: 10.3389/fmed.2020.629086 who are at risk for psychological impairments, such as post-traumatic stress disorder (PTSD), anxiety, and depression. We designed a COVID-19 intensive care unit-specific virtual reality (ICU-VR) intervention and tested it on one of our COVID-19 patients. The impact of event scale-revised and the hospital anxiety and depression scale showed that this patient suffered from PTSD, anxiety, and depression on the day of the intervention. One week after receiving ICU-VR, levels of PTSD, anxiety and depression had normalized, and stayed normalized until 6 months after discharge. In conclusion, innovative technologies, such as VR, have the potential to improve psychological rehabilitation, and should therefore be considered by clinicians for the treatment of ICU-related psychological sequelae after COVID-19.

Keywords: COVID-19, post-intensive care syndrome, critical care, virtual reality, post-traumatic stress disorder, anxiety, depression

\section{INTRODUCTION}

In December 2019, a novel coronavirus, SARS-CoV-2, was first found in Wuhan, China, and rapidly spread around the world. The outbreak has officially been declared a pandemic by the World Health Organization in March 2020 (1). ICU-admission rates were reported as high as 16\% (2). Because a majority of patients treated in the ICU for COVID-19 survive (survival rate in our ICU: 76\%), a large group of ICU survivors can be anticipated.

One-third of general ICU survivors develop psychological impairments, such as post-traumatic stress disorder (PTSD), anxiety, and depression, due to their ICU stay $(3,4)$. These impairments are part of the post-intensive care syndrome (PICS), and cause a decrease in health-related quality of life and a patient's ability to return to their former life. Known risk factors for these impairments are prolonged mechanical ventilation, benzodiazepines, and a prolonged ICU stay (5). These factors were all present during COVID-19 ICU treatment, and clinicians must therefore be prepared for an increase in the incidence of COVID-19 stress-related psychopathological sequelae (6). Due to the large number of COVID-19 ICU-survivors, a uniform, low-time-consuming and easy-to-implement treatment modality is needed.

Virtual reality (VR) is a relatively new technique that has been proven to be effective for treating several psychological impairments, including PTSD and anxiety disorders (7-10). VR has three major advances; first, it represents a means of addressing the limitations of imaginal exposure 
and overcomes the inability to engage in sufficient detail and affective magnitude to recreate the traumatic event, a significant hurdle of imaginal exposure; second, it is an appropriate tool for patient information delivery, and can thus be used to provide additional treatment information, of which post-ICU patients are in need; and third, using VR, one can truthfully reconstruct phases of ICU treatment to replace and adjust possible delusional memories, the largest contributor to psychological distress (1113). As such, Virtual Reality could be a valuable adjunct to improve psychological recovery and health-related quality of life after ICU treatment for COVID-19 $(13,14)$. Here, we describe the effect of an intensive care unit-specific VR intervention in the first patient that visited our COVID-19 outpatient clinic.

\section{MATERIALS AND METHODS}

We developed a COVID-19 intensive care unit-specific virtual reality (ICU-VR) intervention based on previous findings. A similar VR-module was tested safe in healthy volunteers and demonstrated beneficial effects in sepsis survivors (15, 16). In short, the content of ICU-VR was determined by an interdisciplinary team of ICU physicians and nurses, a psychologist, a psychiatrist, and a post-ICU patient. We adapted this module suitable for COVID-19 ICU survivors by adding additional COVID-19 specific aspects of ICU treatment (i.e., mechanical ventilation in prone position, tracheostomy and isolation measures) and information regarding SARS-CoV-2. The COVID-19 ICU-VR intervention thereby consists of six scenes; (1) the ICU physician and nurse welcome the patient in front of the ICU, where the patient is virtually installed in an ICU bed. After being brought to and installed in the ICU room, explanation is given (2) about the surveillance monitor, medication pumps, intubation (including tracheal tube suction), mechanical ventilation, and prone positioning; (3) about the peripheral drip, central line, arterial line, gastric tube, and tracheotomy, including its procedures; (4) about the treatment team and their responsibilities, (5) about isolation measures and personal protection equipment, and (6) about SARS-CoV-2 and COVID-19 (Figures 1A-F).

We tested the module in a COVID-19 patient after ICU treatment. Seven days after hospital discharge, he underwent the ICU-VR intervention twice during his visit to the outpatient clinic. After the first VR session, the patient directly asked to see the VR module for a second time. Hereafter he was given the opportunity to ask questions and to discuss the experience.

The hospital anxiety and depression scale (HADS) and impact of event scale-revised (IES-R) were used to assess anxiety-, depression-, and post-traumatic stress-related symptoms on the day of the COVID-19 outpatient clinic visit, directly prior to receiving the intervention, and 7 days and 6 months after hospital discharge $(17,18)$.

\section{RESULTS}

The patient was male, 57 years old, known with non-insulin dependent diabetes type 2 , severe obstructive apnea syndrome, and no psychiatric history. He was initially admitted for respiratory insufficiency caused by a diffuse bilateral COVID19 pneumonia, after being ill for 5 days. After 2 days of ICU treatment including deep sedation and ventilation in prone positioning, he deteriorated and was transferred to our hospital. After a consecutive treatment of 20 days in our ICU, he underwent a tracheostomy for weaning failure and started weaning in combination with intensive physiotherapy. The patient was eventually discharged from the ICU after 39 days and from the hospital after 54 days.

Seven days after discharge, he received the ICU-VR intervention twice. Prior to receiving ICU-VR, the IES-R sum score was 56, indicating severe PTSD, and the HADS depression and anxiety score were 9 and 13, indicating clinically relevant depression and anxiety, respectively. One week after receiving the COVID-19 specific ICU-VR, the IES-R score was 24, indicating none to mild PTSD, the HADS depression score was one, and the HADS anxiety score was three, indicating neither clinically relevant anxiety nor depression. More importantly, the patient stated that ICU-VR had helped him to better grasp ICU treatment, helped him to put his delusional memories into perspective, and thereby helped him to better cope with ICU-related psychological sequelae. He experienced the intervention as an enervating treatment modality and would recommend it to all patients treated for COVID-19 in the ICU.

Six months after hospital discharge, the patient suffered from critical illness polyneuropathy, had some pulmonary residual abnormalities on CT, but no decline in pulmonary function (FVC: 4.24, 82\%; FEV1: 3.09, 78\%). He did not suffer from psychological impairments with a still normalized IES-R score and HADS anxiety and depression scores. He partially started working again.

\section{DISCUSSION}

This is the first report of intensive care unit-specific virtual reality to reduce psychological distress and improve healthrelated quality of life after ICU treatment for COVID-19. The patient described showed a considerable decrease in posttraumatic stress disorder-, anxiety-, and depression-related symptomatology, shortly after seeing the VR module. Moreover, he reported that the intervention had helped him put his delusional memories into perspective and make sense of what happened to him during ICU treatment.

In the psychology field, VR is becoming an accepted treatment modality for several non-ICU-related psychological disorders (10). Although we are aware that the current report does not provide empirical evidence, it suggests that, especially during the current demand of care, an innovative technique, such as VR, can be considered to improve psychological well-being after ICU treatment. A larger, formally powered, randomized controlled trial should definitely demonstrate the effectiveness of our COVID-19 ICU-VR intervention. We are currently performing such a trial (Netherlands Trial Register, http://www.trialregister. nl/, identifier: NL8835). 

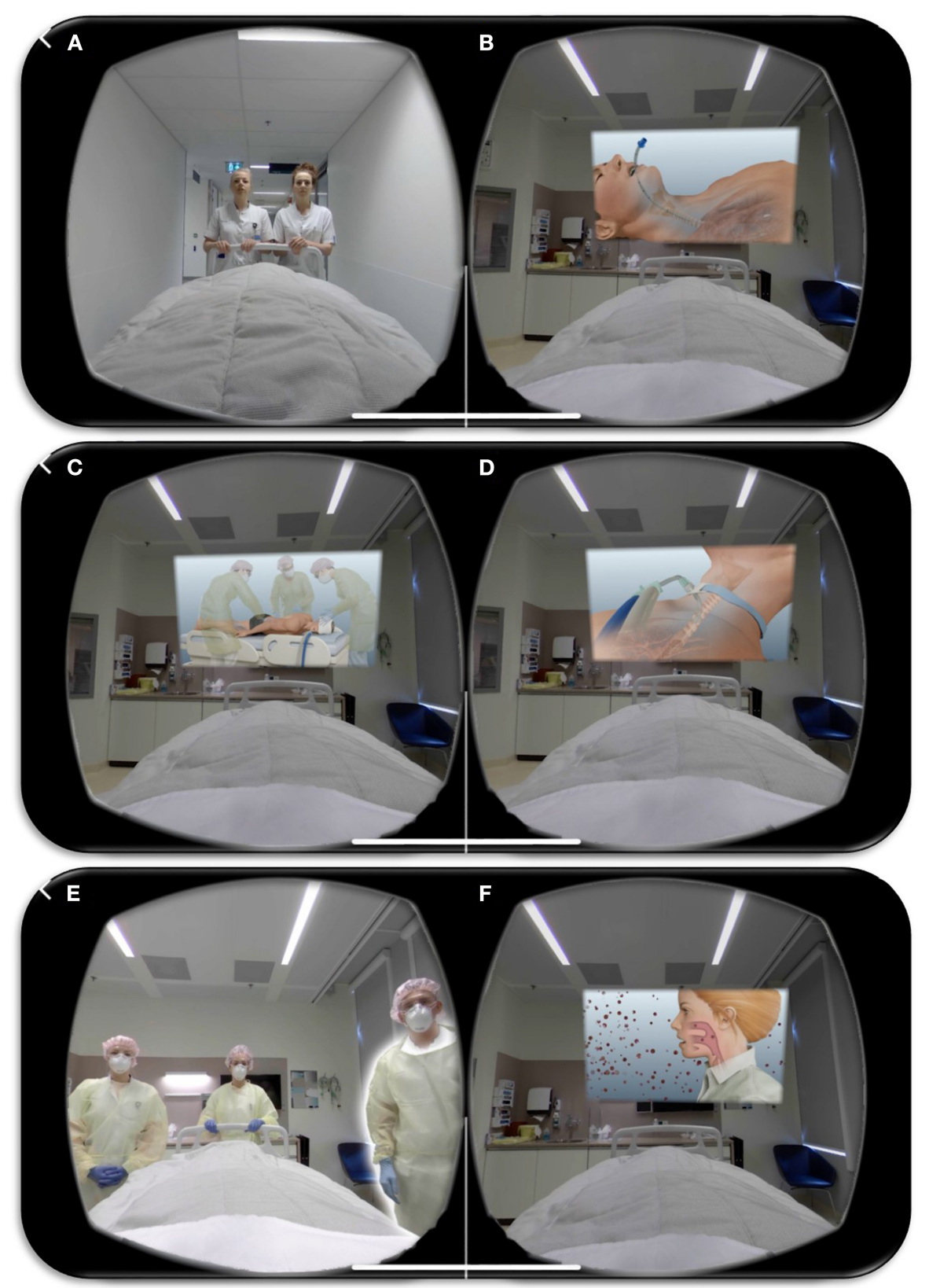

FIGURE 1 | Impression of the COVID-19 Intensive Care Unit-specific Virtual Reality intervention. Screenshots of the COVID-19 Intensive Care Unit-specific Virtual Reality intervention; (1) the ICU physician and nurse welcome the patient in front of the ICU (A), where the patient is virtually installed in an ICU bed. After being brought to and installed in the ICU room, explanation is given about (2) the surveillance monitor, medication pumps, intubation (including trachea tube suction) (B), mechanical ventilation, and prone positioning (C); (3) about the peripheral drip, central line, arterial line, gastric tube, and tracheotomy, including its procedures (D); (4) about the treatment team and their responsibilities, (5) about isolation measures and personal protection equipment (E), and (6) about SARS-CoV-2 and COVID-19 (F).

Anxiety- and stress-related disorders are common in ICU survivors, and literature suggests that these are predominantly caused by delusional memories, sensory overload, and amnesia (19-21). A recent study by Di Nicola et al. added important knowledge and suggested a role of serum 25-hydroxyvitamine $\mathrm{D}$ levels and suggested a role of serum 25-hydroxyvitamine D levels in the development of symptoms of psychological stress
(22). Although the role of serum 25-hydroxyvitamine D in the development of psychological sequelae after ICU treatment has not previously been examined, it could be hypothesized that low serum levels during ICU treatment could influence psychological outcomes. Future studies should examine this hypothesis, and the possibility to prevent psychological PICS by normalizing serum levels of vitamin D. 
It should be acknowledged that we used self-reported questionnaires to assess the patient's psychological well-being. These questionnaires are commonly used in, and accepted for, patients after critical illness and are as such validated in critical care survivors $(23,24)$. One should however take into account that formal assessment of psychological disorders requires a consultation with a psychologist or psychiatrist. Additionally, although the decrease in psychological distress was substantial and can therefore be expected to be at least partially explained by the intervention, we were unable to formally rule out that other factors may have influenced the decrease in psychological sequelae.

In conclusion, innovative technologies, such as VR, have the potential to improve psychological rehabilitation, and should therefore be considered by clinicians for the treatment of ICUrelated psychological sequelae after COVID-19.

\section{DATA AVAILABILITY STATEMENT}

The original contributions presented in the study are included in the article/supplementary material, further inquiries can be directed to the corresponding author/s.

\section{REFERENCES}

1. Wang C, Horby PW, Hayden FG, Gao GF. A novel coronavirus outbreak of global health concern. Lancet. (2020) 395:4703. doi: 10.1016/S0140-6736(20)30185-9

2. Grasselli G, Pesenti A, Cecconi M. Critical care utilization for the COVID19 outbreak in lombardy, italy: early experience and forecast during an emergency response. JAMA. (2020) 323:1545-6. doi: 10.1001/jama.2020.4031

3. Needham DM, Davidson J, Cohen H, Hopkins RO, Weinert C, Wunsch $\mathrm{H}$, et al. Improving long-term outcomes after discharge from intensive care unit: report from a stakeholders' conference. Crit. Care Med. (2012) 40:5029. doi: 10.1097/CCM.0b013e318232da75

4. Bienvenu OJ, Friedman LA, Colantuoni E, Dinglas VD, Sepulveda KA, Mendez-Tellez P, et al. Psychiatric symptoms after acute respiratory distress syndrome: a 5-year longitudinal study. Intensive Care Med. (2018) 44:3847. doi: 10.1007/s00134-017-5009-4

5. Sivanathan L, Wunsch H, Vigod S, Hill A, Pinto R, Scales DC. Mental illness after admission to an intensive care unit. Intensive Care Med. (2019) 45:1550-8. doi: 10.1007/s00134-019-05752-5

6. Rogers JP, Chesney E, Oliver D, Pollak TA, McGuire P, Fusar-Poli $\mathrm{P}$, et al. Psychiatric and neuropsychiatric presentations associated with severe coronavirus infections: a systematic review and meta-analysis with comparison to the COVID-19 pandemic. Lancet Psychiatry. (2020) 7:61127. doi: 10.1016/S2215-0366(20)30203-0

7. Kothgassner OD, Goreis A, Kafka JX, Van Eickels RL, Plener PL, Felnhofer A. Virtual reality exposure therapy for posttraumatic stress disorder (PTSD): a meta-analysis. Eur J Psychotraumatol. (2019) 10:1654782. doi: $10.1080 / 20008198.2019 .1654782$

8. Wechsler TF, Kumpers F, Muhlberger A. Inferiority or even superiority of virtual reality exposure therapy in phobias?-a systematic review and quantitative meta-analysis on randomized controlled trials specifically comparing the efficacy of virtual reality exposure to gold standard in vivo exposure in agoraphobia, specific phobia, social phobia. Front. Psychol. (2019) 10:1758. doi: 10.3389/fpsyg.2019.01758

9. Deng W, Hu D, Xu S, Liu X, Zhao J, Chen Q, et al. The efficacy of virtual reality exposure therapy for PTSD symptoms: A systematic review and meta-analysis. J Affect Disord. (2019) 257:698-709. doi: 10.1016/j.jad.2019. 07.086

\section{ETHICS STATEMENT}

Written informed consent was obtained from the individual(s) for the publication of any potentially identifiable images or data included in this article.

\section{AUTHOR CONTRIBUTIONS}

$\mathrm{JV}, \mathrm{JvB}, \mathrm{E}-\mathrm{JW}$, and $\mathrm{MvG}$ designed the intensive care unit specific virtual reality film. JV, MvG, and $\mathrm{MH}$ recruited the patient. JV and MG wrote the first draft of the manuscript. JvB, MH, E-JW, and DG helped drafting the manuscript. All authors reviewed and approved the final version of the manuscript.

\section{FUNDING}

This project was funded by BeterKeten (foundation), Stichting Coolsingel (foundation), DSW, Stichting Thea (foundation), and Stichting SGS (foundation). The funding sources had no role in writing this manuscript.

10. Park MJ, Kim DJ, Lee U, Na EJ, Jeon HJ. A literature overview of virtual reality (VR) in treatment of psychiatric disorders: recent advances and limitations. Front Psychiatry. (2019) 10:505. doi: 10.3389/fpsyt.2019.00505

11. Bekelis K, Calnan D, Simmons N, MacKenzie TA, Kakoulides G. Effect of an immersive preoperative virtual reality experience on patient reported outcomes: a randomized controlled trial. Ann. Surg. (2017) 265:106873. doi: $10.1097 /$ SLA.0000000000002094

12. Peri T, Gofman M. Narrative Reconstruction: An integrative intervention module for intrusive symptoms in PTSD patients. Psychol Trauma. (2014) 6:176. doi: $10.1037 / \mathrm{a} 0031965$

13. Vlake JH, van Genderen ME, Schut A, Verkade M, E-Wils, Gommers $\mathrm{D}$, et al. Patients suffering from psychological impairments following critical illness are in need of information. J. Intens. Care. (2020) 8:6. doi: 10.1186/s40560-019-0422-0

14. Singh RP, Javaid M, Kataria R, Tyagi M, Haleem A, Suman R. Significant applications of virtual reality for COVID-19 pandemic. Diabetes Metab. Syndr. (2020) 14:661-4. doi: 10.1016/j.dsx.2020.05.011

15. Vlake JH, Wils EJ, Van Bommel J TI.Korevaar MG, Diederik, Van Genderen. ME, Intensive Care Specific Virtual Reality Exposure Therapy is immersive, feasible and safe to treat psychological post-ICU sequelae; a healthy adult study. ICMx. (2019) 7:162-3. doi: 10.1186/s40635-019-0265-y

16. Vlake JH, E-Wils J, Van Bommel JT, Korevaar IM, Gommers D, Van Genderen. ME. Intensive care specific virtual reality (ICU-VR) to improve post-intensive care syndrome-related psychological sequelae in survivors of critical illness. ICMx. (2020) 8:63. doi: 10.1186/s40560-020-00354-8

17. Zigmond AS, Snaith RP. The hospital anxiety and depression scale. Acta Psychiatr Scand. (1983) 67:361-70. doi: 10.1111/j.1600-0447.1983.tb09716.x

18. Weiss DS. The Impact of Event Scale: Revised, Cross-Cultural Assessment of Psychological Trauma and PTSD. Springer (2007). p. 219-38. doi: 10.1007/978-0-387-70990-1_10

19. Askari Hosseini SM, Arab M, Karzari Z, Razban F. Post-traumatic stress disorder in critical illness survivors and its relation to memories of ICU. Nurs Crit Care. (2020) 1-7. doi: 10.1111/nicc.12532

20. Doig L, Solverson K. Wanting to forget: intrusive and delusional memories from critical illness. Case Rep Crit Care. (2020) 2020:7324185. doi: 10.1155/2020/7324185

21. Granja C, Gomes E, Amaro A, Ribeiro O, Jones C, Carneiro A, et al. Understanding posttraumatic stress disorder-related symptoms after critical 
care: the early illness amnesia hypothesis. Crit. Care Med. (2008) 36:28019. doi: 10.1097/CCM.0b013e318186a3e7

22. Di Nicola M, Dattoli L, Moccia L, Pepe M, Janiri D, Fiorillo A, et al. Serum 25-hydroxyvitamin D levels and psychological distress symptoms in patients with affective disorders during the COVID-19 pandemic. Psychoneuroendocrinology. (2020) 122:104869. doi: 10.1016/j.psyneuen.2020. 104869

23. Sukantarat KT, Williamson RC, Brett SJ. Psychological assessment of ICU survivors: a comparison between the hospital anxiety and depression scale and the depression, anxiety and stress scale. Anaesthesia. (2007) 62:239-43. doi: 10.1111/j.1365-2044.2006. 04948.x

24. Bienvenu OJ, Williams JB, Yang A, Hopkins RO, Needham DM. Posttraumatic stress disorder in survivors of acute lung injury: evaluating the Impact of Event Scale-Revised. Chest. (2013) 144:24-31. doi: 10.1378/chest. 12-0908

Conflict of Interest: The authors declare that the research was conducted in the absence of any commercial or financial relationships that could be construed as a potential conflict of interest.

Copyright (ㄷ 2021 Vlake, van Bommel, Hellemons, Wils, Gommers and van Genderen. This is an open-access article distributed under the terms of the Creative Commons Attribution License (CC BY). The use, distribution or reproduction in other forums is permitted, provided the original author(s) and the copyright owner(s) are credited and that the original publication in this journal is cited, in accordance with accepted academic practice. No use, distribution or reproduction is permitted which does not comply with these terms. 